Supporting Information

\title{
Effect of oxygen functional groups in reduced graphene oxide-coated silk electronic textiles for enhancement of $\mathrm{NO}_{2}$ gas sensing performance
}

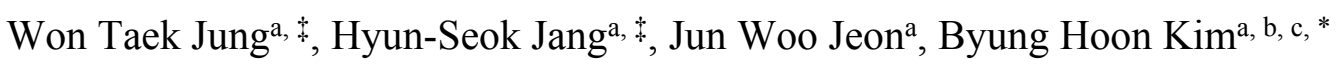

${ }^{a}$ Department of Physics, Incheon National University, Incheon 22012, Republic of Korea

${ }^{b}$ Intelligent Sensor Convergence Research Center, Incheon National University, Incheon 22012, Republic of Korea

c Institute of Basic Science, Incheon National University, Incheon 22012, Republic of Korea

*Corresponding author (kbh37@inu.ac.kr)

$\$$ These authors contributed equally to this work. 
Table S1. The response of the rGO-based e-textile $\mathrm{NO}_{2}$ sensors.

\begin{tabular}{|c|c|c|c|c|c|}
\hline Sample & Temp. $\left({ }^{\circ} \mathrm{C}\right)$ & Condition & $\mathrm{NO}_{2}(\mathrm{ppm})$ & Response (\%) & References \\
\hline rGO/Silk & 25 & Dry & 1.0 & 15.5 & This work \\
\hline rGO/Nylon-6 & 100 & Dry & 1.2 & 14.0 & $\begin{array}{c}\text { Nanoscale } 2014,6 \\
6511-6514\end{array}$ \\
\hline rGO/Cotton Yarn & $\mathrm{RT}$ & Dry & 1.25 & 12.0 & \multirow{2}{*}{$\begin{array}{c}\text { Sci. Rep. } 2015,5 \\
10904\end{array}$} \\
\hline rGO/Polyester Yarn & RT & Dry & 1.25 & 12.0 & \\
\hline rGO/CT/ET Fiber & RT & Dry & 1.0 & 7.0 & $\begin{array}{c}\text { ACS Sens. 2019, 4, } \\
10,2809-2818 \\
\end{array}$ \\
\hline rGO/Cotton Yarn & RT & Dry & 2.5 & $<10.0$ & $\begin{array}{l}\text { Sens. Actuators B- } \\
\text { Chem. 2017, 248, } \\
\quad 829-835\end{array}$ \\
\hline $\begin{array}{c}\text { rGO/Nylon Mesh } \\
\text { Fiber }\end{array}$ & RT & RH $80 \%$ & 1.0 & 13.6 & $\begin{array}{l}\text { Sens. Actuators B- } \\
\text { Chem. 2018, 257, } \\
846-852\end{array}$ \\
\hline rGO/polyester sheet & RT & Dry & 1.0 & 2.75 & $\begin{array}{l}\text { Sens. Actuators B- } \\
\text { Chem. 2021, 345, } \\
130361\end{array}$ \\
\hline rGO coated yarn & RT & RH $25 \%$ & 1.5 & 15.0 & $\begin{array}{l}\text { RSC. Adv. 2018, 8, } \\
\quad 11991-11996\end{array}$ \\
\hline $\begin{array}{l}\text { rGO/polyester, } \\
\text { spandex yarn }\end{array}$ & RT & Dry & 5.0 & $>15$ & $\begin{array}{c}\text { RSC Adv. 2018, } 8, \\
7615-7621\end{array}$ \\
\hline planar rGO sheet & RT & Dry & 1.0 & 3.7 & $\begin{array}{l}\text { ACS Appl. Mater. } \\
\text { Interfaces } 2019 \\
11,9309-9316\end{array}$ \\
\hline rGO thin film & 90 & Dry & 1.0 & $\sim 4$ & $\begin{array}{l}\text { Appl.Surf. Sci. } \\
\text { 2018, 456, 7-12 }\end{array}$ \\
\hline
\end{tabular}
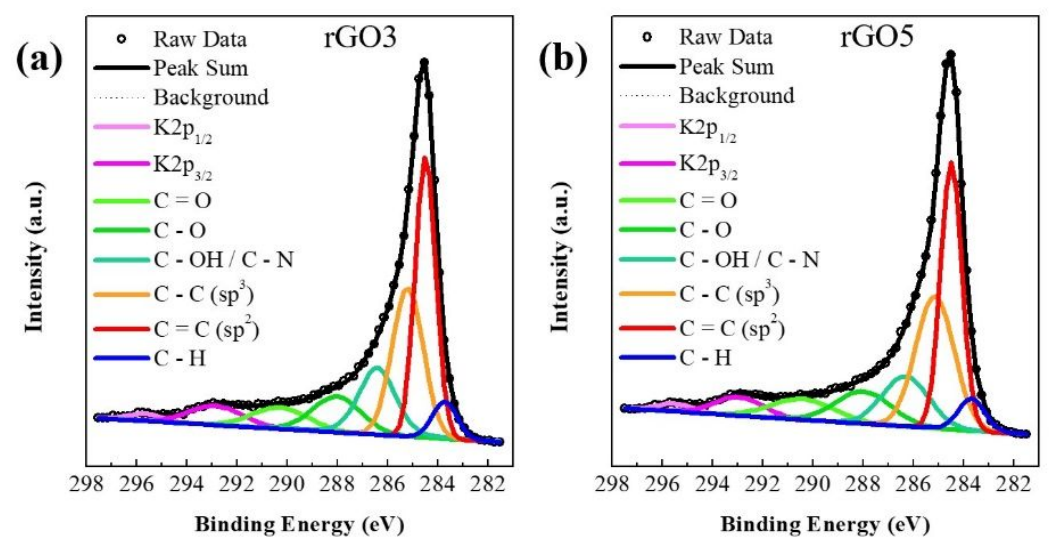

Figure S1. XPS C1s spectra of (a) rGO3 and (b) rGO5. 

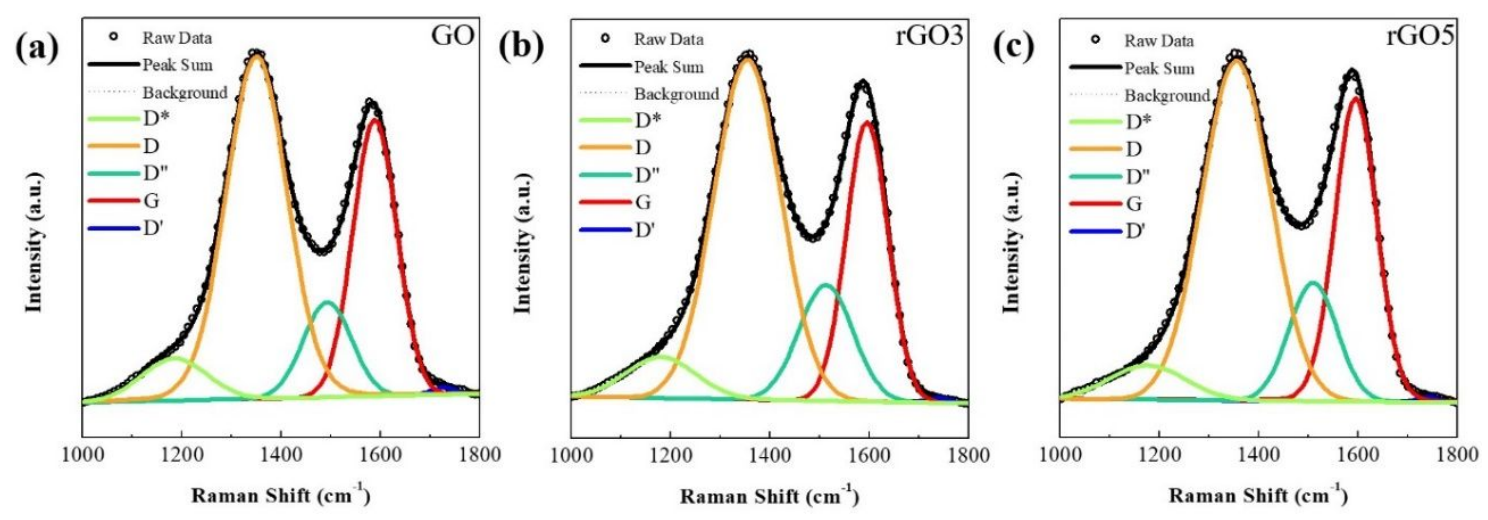

Figure S2. The fitted Raman spectra of (a) GO, (b) rGO3, and (c) rGO5.
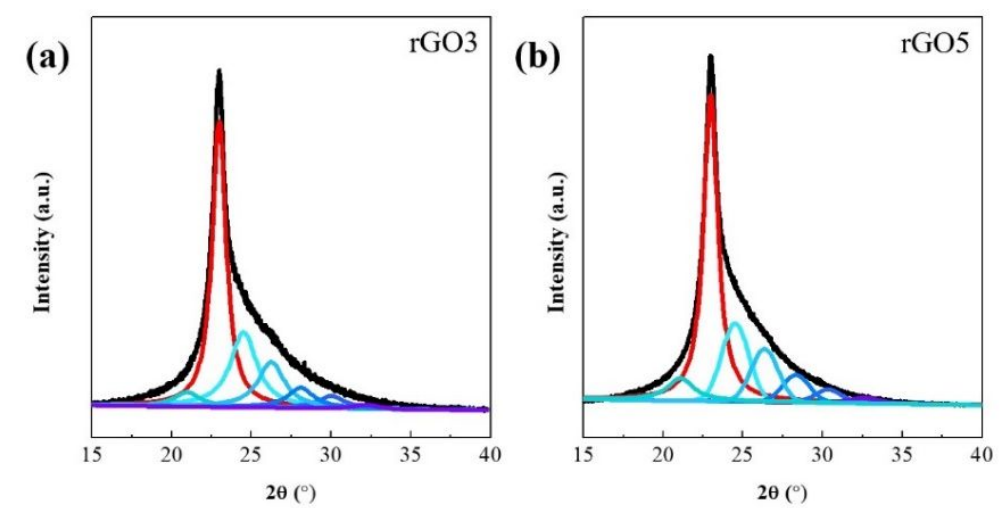

Figure S3. The amorphous structure overlapped (002) lattice plane of (a) rGO3 and (b) rGO5.

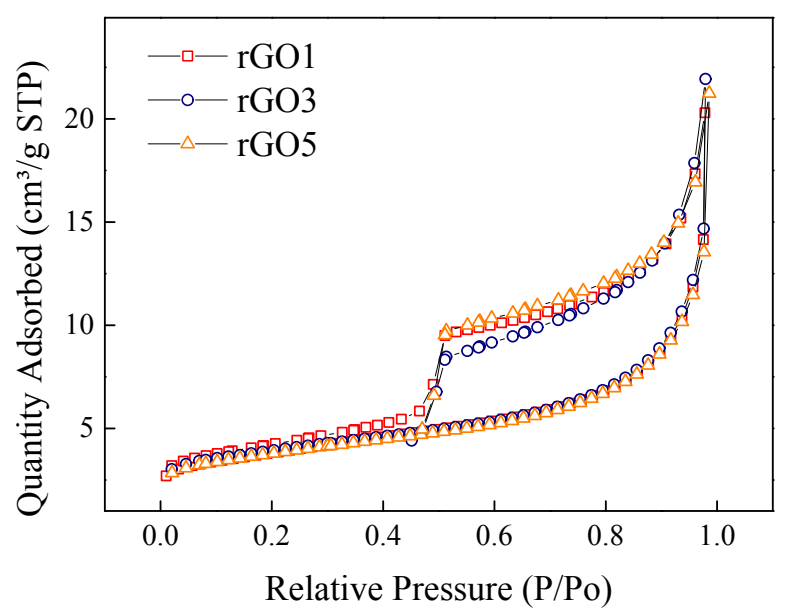

Figure S4. $\mathrm{N}_{2}$ sorption isothermals of rGOn at $77 \mathrm{~K}$. 

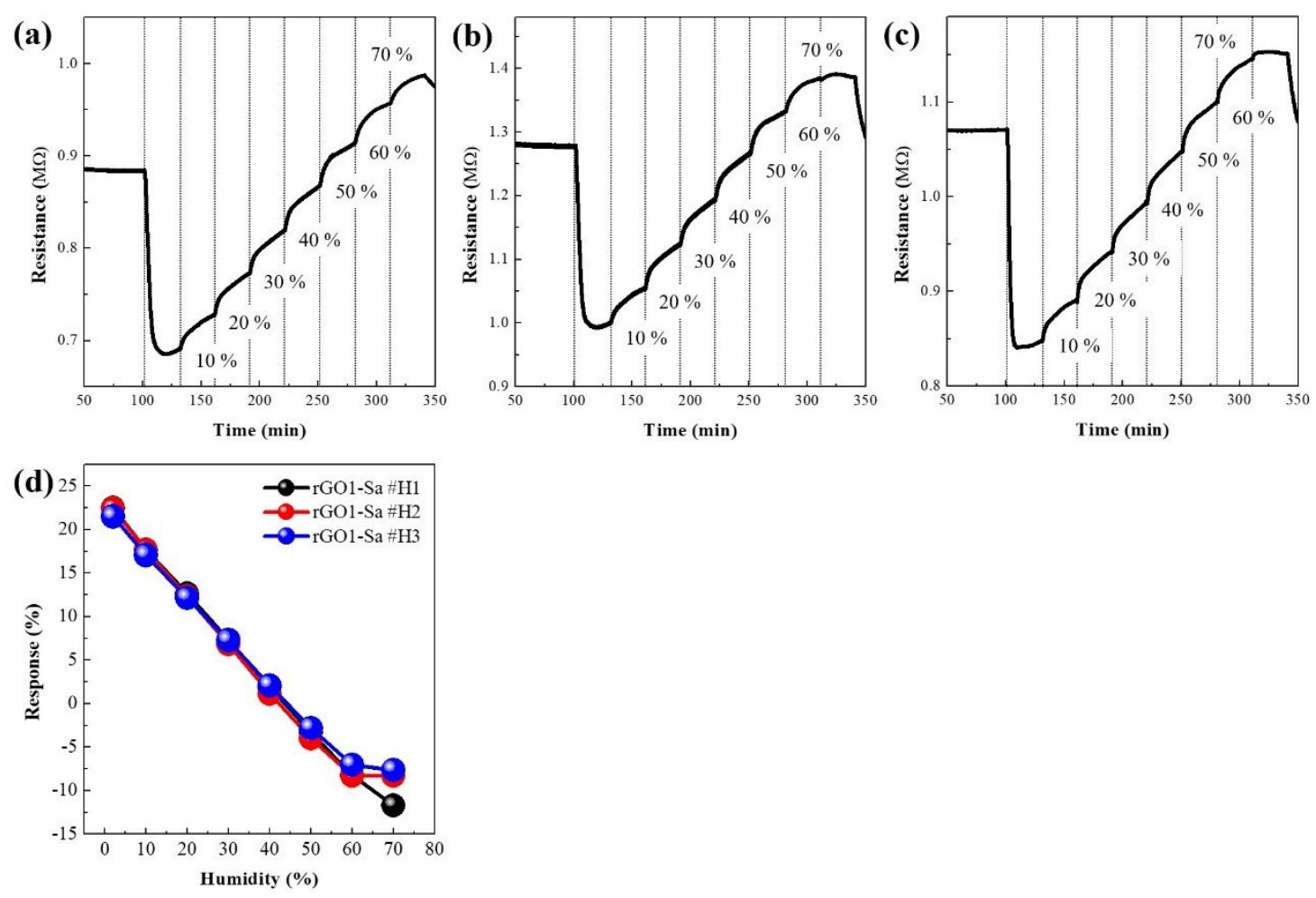

Figure S5. The resistance variance of (a) rGO1-Sa \#H1, (b) rGO1-Sa \#H2, and (c) rGO1-Sa \#H3 reduced heating rate $1{ }^{\circ} \mathrm{C} / \mathrm{min}$. (d) The sensing response of the three rGO1-Sa samples as a function of humidity.

\section{References}

[S1] Yun, Y. J.; Hong, W. G.; Choi, N. -J.; Park, H. J.; Moon, S. E.; Kim, B. H.; Song, K. B.; Jun, Y.; Lee, H. -K. A 3D scaffold for ultra-sensitive reduced graphene oxide gas sensors. Nanoscale. 2014 ,6, 6511-6514.

[S2] Yun, Y. J.; Hong, W. G.; Choi, N. -J.; Kim, B. H.; Jun, Y.; Lee, H. -K. Ultrasensitive and highly selective Graphene-based single yarn for use in wearable gas sensor. Sci. Rep. 2015, $5,10904$. 
[S3] Li, W.; Chen, R.; Qi, W.; Cai, Li.; Sun, Y.; Sun, M.; Li, C.; Yang, X.; Xiang, L.; Xie, D.; Rne, T. Reduced Graphene Oxide/Mesoporous ZnO NSs Hybrid Fibers for Flexible, Stretchable, Twisted, and Wearable $\mathrm{NO}_{2}$ E-Textile Gas Sensor. ACS Sens. 2019, 4, 10, 28092818.

[S4] Yun, Y. J.; Hong, W. G.; Kim, D. Y.; Kim, H. J., Jun, Y.; Lee, H. -K. E-textile gas sensors composed of molybdenum disulfide and reduced graphene oxide for high response and reliability. Sens. Actuators B-Chem. 2017, 248, 829-835.

[S5] Park, H. J.; Kim, W. -J.; Lee, H. -K.; Lee, D. -S.; Shin, J. -H.; Jun, Y.; Yun, Y. J. Highly flexible, mechanically stable, and sensitive $\mathrm{NO}_{2}$ gas sensors based on reduced graphene oxide nanofibrous mesh fabric for flexible electronics. Sens. Actuators B-Chem. 2018, 257, 846-852.

[S6] Lee, S. W.; Jung, H. G.; Jang, J. W.; Park, D.; Lee, D.; Kim, I.; Kim, Y.; Cheong, D. Y.; Hwang, K. S.; Lee, G.; Yoon, D. S. Graphene-based electronic textile sheet for highly sensitive detection of $\mathrm{NO}_{2}$ and $\mathrm{NH}_{3}$. Sens. Actuators B-Chem. 2021, 345, 130361.

[S7] Kang, M. -A.; Ji, S.; Kim, S.; Park, C. -Y.; Myung, S.; Song, W.; Lee, S. S.; Lim, J.; An, K. -S. Highly sensitive and wearable gas sensors consisting of chemically functionalized graphene oxide assembled on cotton yarn. $R S C A d v . \mathbf{2 0 1 8}, 8,11991-11996$.

[S8] Yun, Y. J.; Kim, D. Y.; Hone, W. G.; Ha, D. H.; Jun, Y.; Lee, H. -K. Highly stretchable, mechanically stable, and weavable reduced graphene oxide yarn with high $\mathrm{NO}_{2}$ sensitivity for wearable gas sensors. $R S C A d v$. 2018, 8, 7615-7621.

[S9] Li, F.; Peng, H.; Xia, D.; Yang, J.; Yang, K.; Yin, F.; Yuan, W. Highly Sensitive, Selective, and Flexible $\mathrm{NO}_{2}$ Chemiresistors Based on Multilevel Structured Three-Dimensional 
Reduced Graphene Oxide Fiber Scaffold Modified with Aminoanthroquinone Moieties and Ag Nanoparticles. ACS Appl. Mater. Interfaces 2019, 11, 9, 9309-9316.

[S10] Jung, M. W.; Kang, S. M.; Nam, K. -H.; An, K. -S.; Ku, B. -C. Highly transparent and flexible $\mathrm{NO}_{2}$ gas sensor film based on $\mathrm{MoS}_{2} / \mathrm{rGO}$ composites using soft lithographic patterning. Appl. Surf. Sci. 2018, 456, 7-12. 